\title{
The Synthesis of a Novel Cellulose Physical Gel
}

\author{
Jiufang Duan, ${ }^{1}$ Xiaojian Zhang, ${ }^{1}$ Jianxin Jiang, ${ }^{1}$ Chunrui Han, ${ }^{1}$ Jun Yang, \\ Liujun Liu, ${ }^{1}$ Hongyun Lan, ${ }^{2}$ and Daozhan Huang ${ }^{2}$ \\ ${ }^{1}$ Institute of Material Science and Technology, Beijing University of Forestry, Beijing 100083, China \\ ${ }^{2}$ Guangxi Key Laboratory of Chemistry and Engineering of Forest Products, Guangxi University for Nationalities, \\ Nanning 530006, China
}

Correspondence should be addressed to Jiufang Duan; duanjiu99@163.com

Received 25 November 2013; Revised 27 January 2014; Accepted 29 January 2014; Published 3 March 2014

Academic Editor: Wen Zeng

Copyright (C) 2014 Jiufang Duan et al. This is an open access article distributed under the Creative Commons Attribution License, which permits unrestricted use, distribution, and reproduction in any medium, provided the original work is properly cited.

Cellulose possessing $\beta$-cyclodextrin ( $\beta$-CD) was used as a host molecule and cellulose possessing ferrocene $(\mathrm{Fc})$ as a guest polymer. Infrared spectra, differential scanning calorimetry (DSC), ultraviolet spectroscopy (UV), and contact angle analysis were used to characterise the material structure and the inclusion behaviour. The results showed that the $\beta$-CD-cellulose and the Fc-cellulose can form inclusion complexes. Moreover, ferrocene oxidation, and reduction of state can be adjusted by sodium hypochlorite $(\mathrm{NaClO})$ as an oxidant and glutathione (GSH) as a reductant. In this study, a physical gel based on $\beta$-CD-cellulose/Fc-cellulose was formed under mild conditions in which autonomous healing between cut surfaces occurred after 24 hours. The physical gel can be controlled in the sol-gel transition. The compressive strength of the Fc-cellulose/ $\beta$-CD-cellulose gel increased with increased cellulose concentration. The host-guest interaction between the side chains of cellulose could strengthen the gel. The cellulose physical gel may eventually be used as a stimulus-responsive, healing material in biomedical applications.

\section{Introduction}

In light of heightened environmental and energy concerns, cellulose based on nature biomass has been receiving increasing attention in recent years. Cellulose is the most abundant naturally occurring polymer of glucose, found as the main constituent of plants and natural fibres such as cotton and linen and is considered a renewable and sustainable resource of raw material to satisfy the increasing demand for environmentally friendly [1] and energy-generating products $[2,3]$. Cellulose-based hydrogels are biocompatible and biodegradable materials which show promise for a number of industrial uses, especially in cases where environmental issues are important. Such natural polysaccharides have been widely used for the preparation of gels in various fields [4-7], for example: wastewater treatment, the food industry, cosmetics, biomedical, pharmaceutical, and tissue engineering applications, due to their unique properties such as high swelling capacity, biocompatibility, biodegradability, and biological functions.
Cellulose, th cellulose, the most abundant renewable polysaccharide on earth, is a strong candidate for the fabrication of gels and cellulose-based gels have been reported including cellulose-polymer composite gels and celluloseinorganic hybrid gels. The design and use of cellulose-based hydrogels, which usually couple their biodegradability with a smart stimuli-sensitive behaviour, together with the large availability of cellulose in nature and the low cost of cellulose derivatives, make cellulose-based hydrogels particularly attractive.

Cellulose-based gels $[8,9]$ can be obtained by either physical or chemical stabilisation of aqueous solutions of cellulosics [10-14]. Cellulose-based gels, either reversible or stable, can be formed by properly cross-linking aqueous solutions of cellulose ethers $[12,15,16]$, such as methylcellulose, hydroxypropyl methylcellulose, ethyl cellulose (EC), hydroxyethyl cellulose (HEC), and sodium carboxymethylcellulose (NaCMC), which are among the most widely used cellulose derivatives. Depending on the cellulose derivative used, a number of cross-linking agents and catalysts can be used 
to form gels. Epichlorohydrin, aldehydes, aldehyde-based reagents, urea derivatives, carbodiimides, and multifunctional carboxylic acids are the most widely used cross-linking agents for cellulose [17-21]. In light of material application concerns, cellulose gels have received increasing attention in recent years $[22,23]$. Cellulose supramolecular materials linked by noncovalent bondshave attracted much more attention and have been widely studied [24]; their responsiveness to the external environment [25], such as temperature [26], $\mathrm{pH}$ [27], and medicine (drug release carriers) [28] have been of particular interest.

However, there are few reports on the application of cellulose gel used in healing. In this study, a novel cellulose physical gel was synthesised by $\beta$-CD-cellulose and $\mathrm{Fc}_{\mathrm{c}}$ cellulose. The host-guest interaction between the $\beta$-CDcellulose and the Fc-cellulose, the sol-gel transition, and the redox stimuli properties of the supramolecular material were also studied.

\section{Experimental}

2.1. Material and Methods. Cellulose, $\beta$-cyclodextrin, epichlorohydrin, sodium hydroxide, lithium chloride, N,Ndimethyl acetamide, methylene chloride, ferrocene carboxylic acid, oxalyl chloride, sodium hypochlorite and glutathione were commercially available and used as received.

IR spectra were recorded by FTIR (Nicolet iN10 Thermo Fisher Scientific China) in the region of $400-4000 \mathrm{~cm}^{-1}$. DSC measurements were carried out using a Diamond DSC apparatus (NETZSCHDSC 204). The dried samples were placed in pressure tight aluminum DSC cells from 20 to $150^{\circ} \mathrm{C}$ with a heating rate of $10^{\circ} \mathrm{C} \mathrm{min}{ }^{-1}$. Surface contact angles were measured using a dynamic contact angle analyzer (HARKESPCA, error value of $\pm 0.1^{\circ}$, Beijing HARKE Experimental Instrument Factory). The cellulose powder samples were compressed using an infrared tablet. Surface contact angles were measured during liquid exposure to the test materials for $1 \mathrm{~s}$ in order to avoid material penetration factors as far as possible. The morphological characterization of gel was performed with scanning electron microscopy (S-3400N, HIACHI, Japan).

The gravimetric method was employed to measure the swelling ratios of the gels in distilled water at $25^{\circ} \mathrm{C}$. After immersion in distilled water for about $48 \mathrm{hr}$ to reach swelling equilibrium, the gel samples were taken out and weighed after removing the excess water on the surfaces. Each data was measured three samples, and the average value of three measurements was taken. The equilibrium swelling ratio (SR) was calculated as $\mathrm{SR}=W_{s} / W_{d}$, where $W_{s}$ is the weight of the swollen gel and $W_{d}$ is the weight of the gel at the dry state.

Reswelling ratios of the gels were measured as follows: the dry gel was placed in deionized water of $25^{\circ} \mathrm{C}$. At predetermined time, the gel samples were taken out from the aqueous solution and weighed the quality $W_{t}$, until it reached swelling equilibrium. Reswelling rate $\left(r_{\mathrm{SR}}\right): r_{\mathrm{SR}}=100 *\left(W_{t}-\right.$ $\left.W_{d}\right) / m_{d}$.

\subsection{Preparation of Cellulose Inclusion Complexes}

2.2.1. Preparation of Cellulose-CDs. Cellulose was dissolved in sodium hydroxide/urea. To this solution, cyclodextrin and epichlorohydrin were added. After stirring for $2 \mathrm{~h}, \beta$-CD was added and the solution was stirred for another $12 \mathrm{~h}$ at room temperature. The polymer product was reprecipitated from distilled water and washed with distilled water. The degree of substitution is $0.32 \mathrm{wt} \%$, which was detected by the phenolphthalein probe method [29].

In $\mathrm{NaOH}$ aqueous solution, cellulose and epichlorohydrin generate the epoxy cellulose, which connected hydroxy of $\beta$-cyclodextrin by the epoxy group as a cross-linking bridge.

2.2.2. Synthesis of $\mathrm{Fc}$-COCl. Ferrocenecarboxylic acid was suspended in dichloromethane (DCM). Then oxalyl chloride was added dropwise, and the suspension was stirred for $3 \mathrm{~h}$ at room temperature. The orange suspension turned into a red solution. After evaporating the solvent, the solid product was collected.

2.2.3. Synthesis of Cellulose-Fc. Cellulose was dissolved in lithium chloride/dimethylacetamide (LiCl/DMAc). The ferrocenecarboxylic chloride solution was added dropwise. After stirring overnight at room temperature, the solution was washed with distilled water. The orange solid was washed with lithium chloride; the solid product was collected via a centrifuge and dried for 4 days at $50^{\circ} \mathrm{C}$ to obtain cellulose$\mathrm{Fc}$ as a yellow powder. The ferrocene grafting rate is 0.57 $5.7 \mathrm{wt} \%$, which was detected by weighing before and after the cellulose reaction.

2.2.4. Preparation of Redox Inclusion Complex. The oxidized state (or educed state) products were prepared by shaking appropriate amounts of $\mathrm{NaClO}$ aq. (14 mM) (or GSH) and Fc-CD-cellulose inclusion complexes (or oxidized state products) at room temperature for $24 \mathrm{~h}$. The solution was washed with distilled water and then the solid product was collected via a centrifuge and freeze-dried. A complex mixture was prepared by grinding powders for $20 \mathrm{~min}$.

2.2.5. Synthesis of Cellulose Gel. The ferrocene-cellulose and cyclodextrin-cellulose were dissolved in N,N-Dimethylformamide (DMF) lithium chloride solution. We poured the solution into a glass mould in a humid box until the gel was stabilized.

\section{Results and Discussion}

3.1. Characterization. Figure 1 (curve (a)) and Figure 1 (curve (b)) show the infrared spectra of the cellulose and $\beta$-CDcellulose. According to Figure 1 (curves (a) and (b)), absorption was observed at $3380 \mathrm{~cm}^{-1}$ (hydroxyl stretch influenced by hydrogen bonding), $1646 \mathrm{~cm}^{-1}$ and $1354 \mathrm{~cm}^{-1}$ (carbonyl stretch), $1043 \mathrm{~cm}^{-1}$ (carboxyl in ethers), and $2920 \mathrm{~cm}^{-1}$ (methylene), which were characteristic absorptions in cellulose and $\beta$-CD-cellulose structures. As seen from Figure 1 


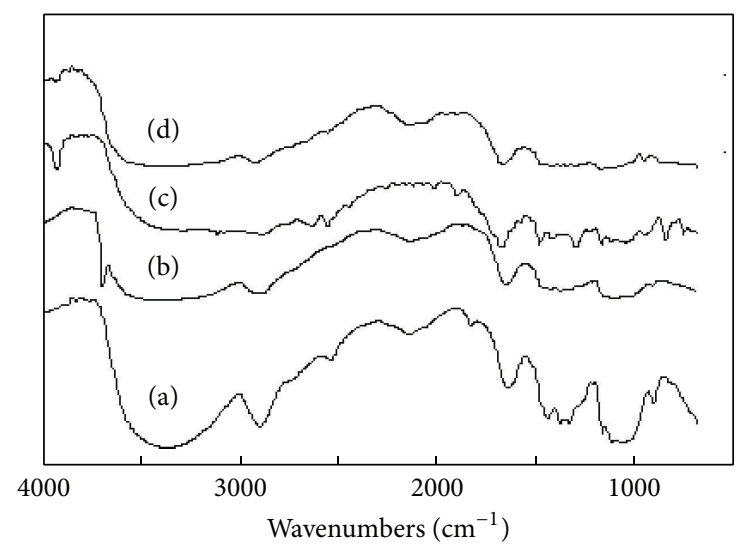

FIGURE 1: Infrared spectra of cellulose ((a) cellulose, (b) cellulose$\mathrm{CD}$, (c) cellulose-Fc, and (d) cellulose-CD-Fc inclusion).

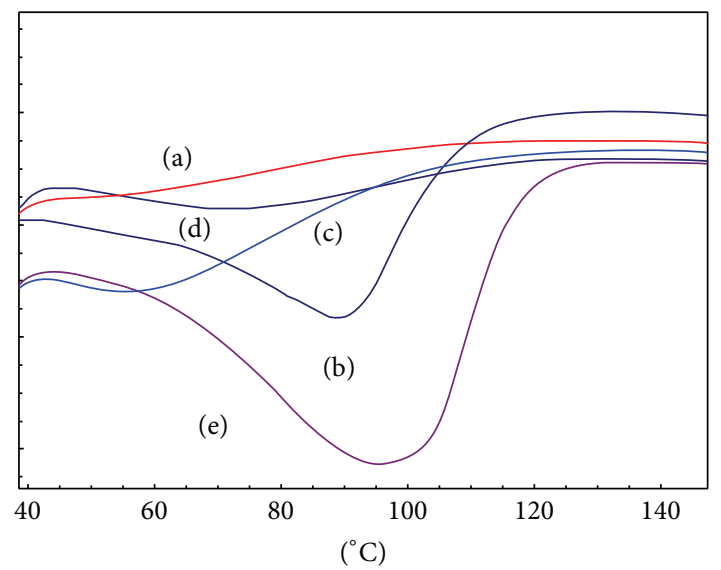

Figure 2: DSC curves of the series of cellulose ((a) cellulose, (b) cellulose-CD, (c) cellulose-ferrocene, (d) cellulose-CD-ferrocene inclusion, (e) simple mixing of cellulose-ferrocene, and celluloseCD).

(curve (c)) the characteristic peaks of ferrocene-cellulose were $1402 \mathrm{~cm}^{-1}, 1100 \mathrm{~cm}^{-1} \mathrm{VC}-\mathrm{C}$ (cyclopentadienyl ring), and $816 \mathrm{~cm}^{-1}$ DC-H (cyclopentadienyl ring). The characteristic peaks of ferrocene disappeared from the infrared spectra of the envelope of the inclusion compound (curve (d)). This indicated that the ferrocene-cellulose and cyclodextrin cellulose have been formed in the inclusion complex. Similar findings were reported in the literature $[30,31]$.

3.2. Thermal Analysis. The thermal analysis curves of the cellulosic inclusion complexes are shown in Figure 2 where it can be seen that the curve (a) of cellulose is almost a straight line, but the curves (b) (CD-cellulose) and (e) (simple physical mixing of CD-cellulose and Fc-cellulose) had a strong exothermic peak (cyclodextrin dehydration absorption peaks [32] at $88.9^{\circ} \mathrm{C}$ and $95.9^{\circ} \mathrm{C}$, resp.), corresponding to the loss of water from the $\mathrm{CD}$ cavity to atmosphere, indicating that $\mathrm{CD}$ was free from guest complexation in the physical mixture [32]. Otherwise, the curve (d) (the inclusion complex) showed a lack of endothermic peaks, which suggested that a different molecule was present in the CD cavity, instead of the water molecules and a lack of pure $\mathrm{CD}$ in the complex sample. Similar results were reported in the literature for interactions between other drugs and CDs $[30,31,33,34]$.

3.3. The Redox Properties. The redox regulation process of inclusion complexes containing ferrocene has been studied extensively.

The host-guest interaction between the metal ferrocene with $\beta$-CD can also be a reversible regulation by oxidation and reduction of the ferrocene. More than 20 years ago, it was reported that the reduced ferrocene effectively forms an inclusion compound with the $\beta$-CD, while the oxidation of the ferrocene was impossible $[35,36]$. The nature of ferrocene is hydrophobic in its reduced state and hydrophilic in its oxidized state. The reversible regulation of the inclusion complexes forming a binary complex can be achieved by changing the redox state of the ferrocene. Therefore, the cellulose materials were expected to have a redox response performance by grafting ferrocene onto the cellulose.

The host-guest interaction between the metal ferrocene with $\beta$-CD can also be a reversible regulation by oxidation and reduction of the ferrocene. More than 20 years ago, it was reported that the ferrocene reduced forms of the inclusion compound with the $\beta$-CD effectively, while the ferrocene oxidized is basically impossible $[35,36]$. The nature of ferrocene is hydrophobic in the reduced state and hydrophilic in the oxidized state. The reversible regulation of the inclusion complexes form of binary complex can be achieved by changing the redox state of the ferrocene. Therefore, the cellulose materials are expected to have the redox response performance by grafting ferrocene on the cellulose.

The redox behaviour of ferrocene was studied by measuring the surface contact angle with water. The cyclodextrincellulose contact angle was $59.6^{\circ}$ (Figure 3(a)) and the ferrocene-cellulose contact angle was $82.1^{\circ}$ (Figure 3(b)). The contact angle changed from $82.1^{\circ}$ to $61.2^{\circ}$ (Figure 3(c)) when the ferrocene-cellulose and cyclodextrin-cellulose formed inclusion complexes. This demonstrated that the ferrocene was an inclusion in the cyclodextrin cavity.

Aqueous $\mathrm{NaClO}$ was chosen as an oxidant and GSH as a reductant. Adding aqueous $\mathrm{NaClO}$ to the cellulose$\beta \mathrm{CD} /$ cellulose-Fc inclusion complexes increased the contact angle from $61.2^{\circ}$ to $71.7^{\circ}$ (Figure $3(\mathrm{~d})$ ). In contrast, continuous addition of GSH to the inclusion complexes recovered the Fc group, causing the contact angle to revert to its former value (Figure 3(e)). Cellulose- $\beta$-CD showed a high affinity for the reduced state of the Fc group due to its hydrophobic nature, whereas the oxidized state of the Fc group $(\mathrm{Fc}+)$ exhibited a low affinity for cellulose- $\beta$-CD due to the cationic $\mathrm{Fc}+$ group [35-37]. Moreover, the cellulose inclusion complexes exhibited excellent lock and unlock properties controlled by the redox of ferrocene.

3.4. The Compressive Strength of $F c$-Cellulose/ $\beta$-CD-Cellulose $\mathrm{Gel}$. Gels are composed of a three-dimensional hydrophilic 


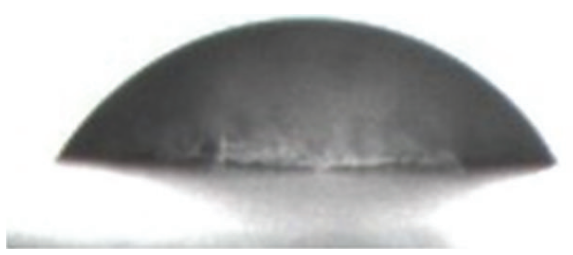

(a)

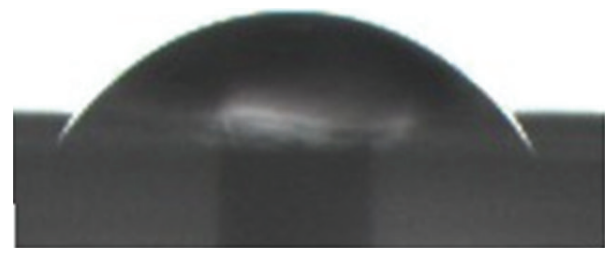

(c)

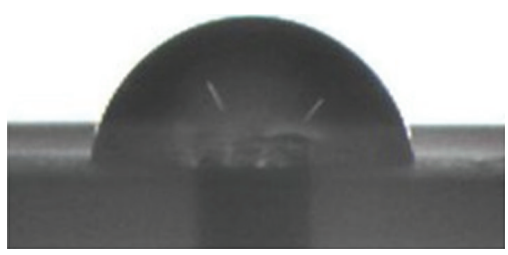

(b)

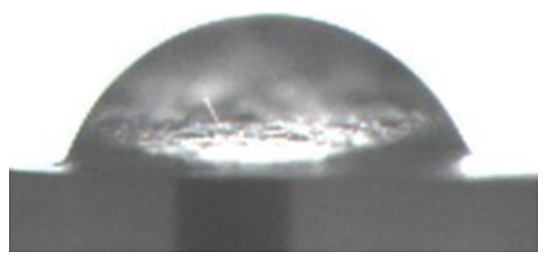

(d)

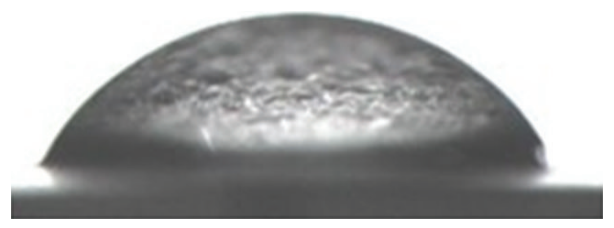

(e)

FIGURE 3: Contact angle images of the series of cellulose ((a) cellulose-CD, (b) cellulose-ferrocene, (c) cellulose-CD-ferrocene inclusion, (d) $\mathrm{NaClO}$ treated sample, and (e) GSH treated sample).

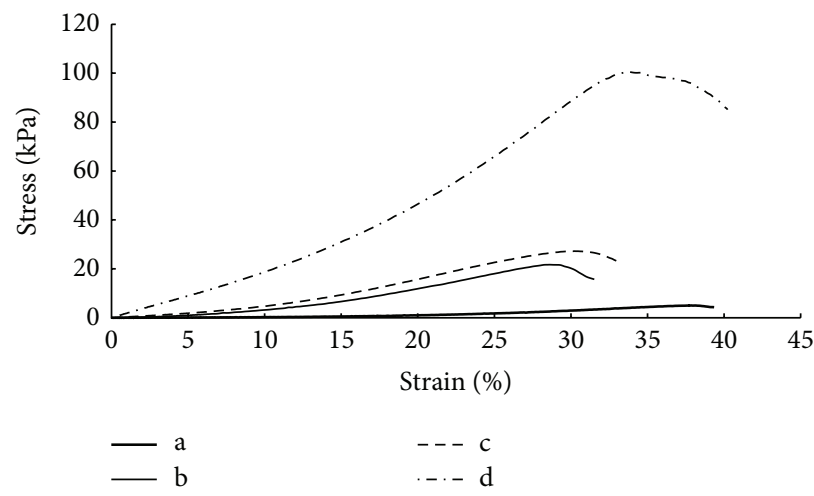

FIgURE 4: The compressive strength of different cellulose content ((a) $1 \mathrm{wt} \%$, (b) $3 \mathrm{wt} \%$, (c) $4 \mathrm{wt} \%$, and (d) $5 \mathrm{wt} \%$ ).

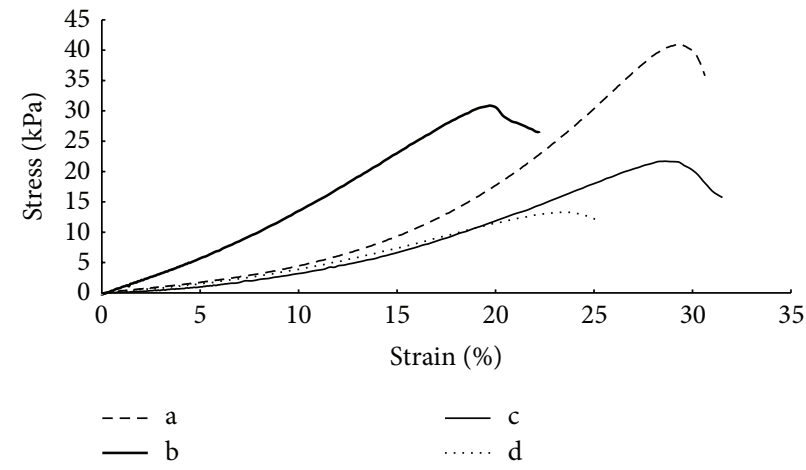

Figure 5: The compressive strength of different grafting ratio of ferrocene ((a) $5.66 \mathrm{wt} \%$, (b) $2.83 \mathrm{wt} \%$, (c) $1.41 \mathrm{wt} \%$, (d) $0.57 \mathrm{wt} \%$ ). 


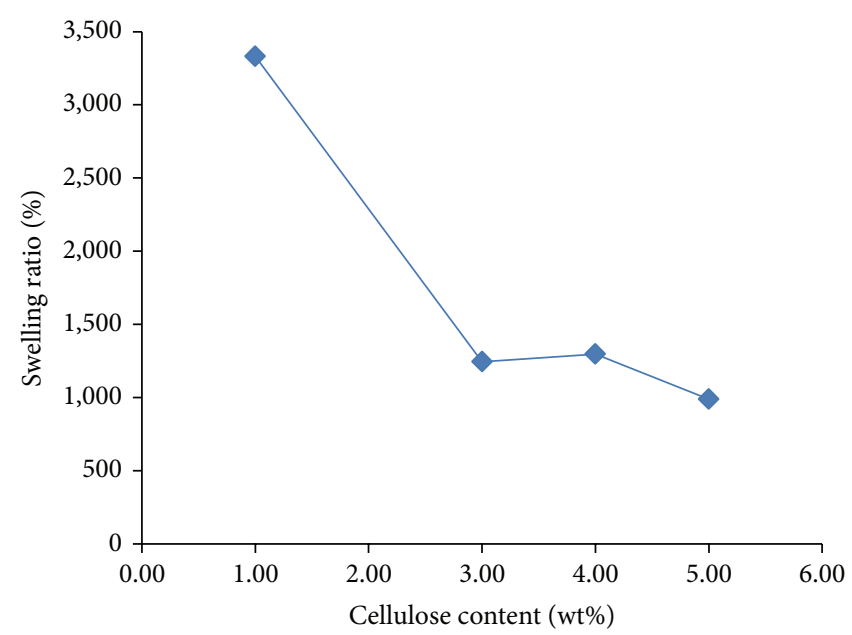

FIGURE 6: The swelling ratio of the Fc-cellulose/ $\beta$-CD-cellulose hydrogel.

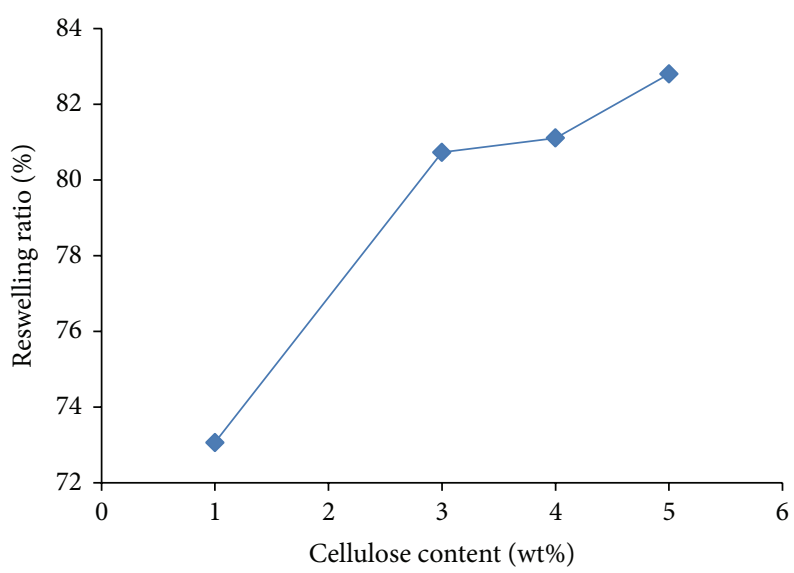

FIGURE 7: The reswelling ratio of the Fc-cellulose/ $\beta$-CD-cellulose hydrogel.

polymer network in which a large amount of water is interposed. The cross-linking ratio of the network is important to the mechanical properties. As the concentration of cellulose increased from $1 \%(\mathrm{w} / \mathrm{w})$ to $5 \%(\mathrm{w} / \mathrm{w})$ the gel strength increased from $5 \mathrm{kPa}$ to $100.5 \mathrm{kPa}$ (Figure 4). This was because the number of cross-linking sites per unit volume of the network increased with an increased number of functional groups on the molecular chain which was caused by the increased concentration of cellulose [38]. Similar improvements in the mechanical strength were also observed when increasing the cross-linking degree by changing the grafting ratio of Fc. The compressive strength of Fc-cellulose/ $\beta$-CDcellulose gel changed from $13.32 \mathrm{kPa}$ to $40.97 \mathrm{kPa}$ as the grafting ratio of Fc changed from $0.57 \%(\mathrm{w} / \mathrm{w})$ to $5.66 \%(\mathrm{w} / \mathrm{w})$ (Figure 5), indicating that the host-guest interaction between the side chains of cellulose participated in the formation of the three-dimensional network structure of the gel and also affected its strength.

3.5. The Water Absorption of Fc-Cellulose/ $\beta$-CD-Cellulose $\mathrm{Gel}$. The swelling ratio of the gel was the most important variable to be evaluated for given environmental conditions, as it affected the diffusive, mechanical, optical, acoustic, and surface properties of the gel itself. The swelling ratio of the Fc-cellulose/ $\beta$-CD-cellulose gel decreased as the concentration of cellulose increased (Figure 6). This was because the amount of water retained by the mesh of the hydrogel network depended on the structure of the polymer network itself [39]. The Fc-cellulose/ $\beta$-CD-cellulose gel was formed by properly cross-linking the cellulose chains. The number of cross-linking sites per unit volume of the polymer network increased with increasing cellulose concentration. The larger degree of cross-linking was not conducive to the diffusion of water molecules.

The reswelling ratio of the Fc-cellulose/ $\beta$-CD-cellulose gel decreased from $3330 \%$ (w/w) (Figure 6) to $73.06 \%(w / w)$ (Figure 7), when the dry gel was placed in deionised water again. The pore sizes of the super-absorbent gel showed that it was the water retention capacity that engendered the efficiency of absorption. It can be observed that the larger number of pores can retain more water. Figure 8 shows the freeze-drying morphology of the cellulose gel and Fccellulose/ $\beta$-CD-cellulose gel; compared with the cellulose gel, 


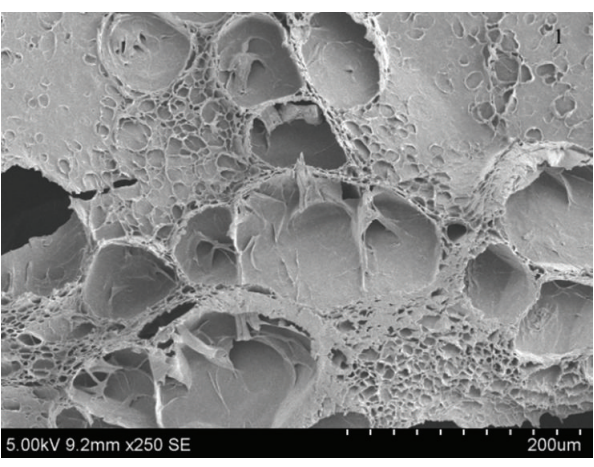

(a)

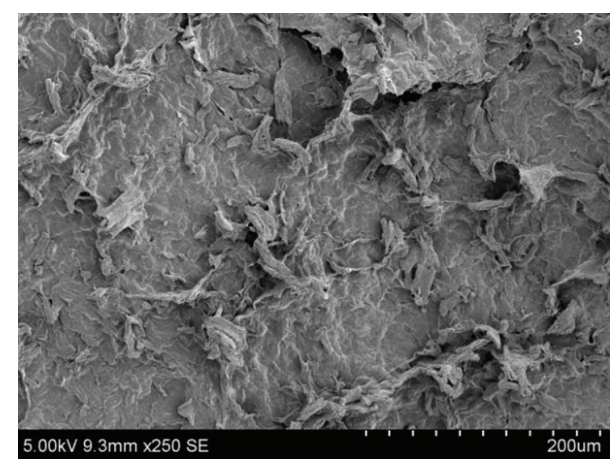

(b)

FIGURE 8: The SEM images of gel ((a) cellulose hydrogel and (b) Fc-cellulose/ $\beta$-CD-cellulose hydrogel).

the Fc-cellulose/ $\beta$-CD-cellulose gel had a more dense pore structure. After drying the gel, the dense pore structure was easily collapsed and confined; therefore, the reswelling ratio of the Fc-cellulose/ $\beta$-CD-cellulose gel was poor.

3.6. The Surface Morphology of the Gel. The surface morphology of the gel is also shown in Figure 8. Cellulose and Fc-cellulose/ $\beta$-CD-cellulose inclusion complexes gels were prepared by the same method. Cellulose gel (Figure 8(a)) had a relatively large, porous structure; as well as the Fccellulose/ $\beta$-CD-cellulose inclusion complexes, the gel had a relatively dense surface structure. This may have been caused by the different intermolecular forces arising from the alteration of the cellulose molecular chain structure.

\section{Conclusions}

Ferrocene and cyclodextrin were grafted onto the cellulose surface, respectively. The cellulose physical gel was prepared by $\mathrm{Fc}$-cellulose and $\beta$-CD-cellulose at room temperature. The $\beta$-CD-cellulose and the Fc-cellulose can form inclusion complexes. Moreover, ferrocene oxidation and reduction of state can be adjusted by sodium hypochlorite $(\mathrm{NaClO})$ as an oxidant and glutathione (GSH) as a reductant. The solgel transition can be controlled. The compressive strength of Fc-cellulose/ $\beta$-CD-cellulose gel increased with increasing cellulose concentration. The host-guest interaction between the side chains of cellulose can strengthen the gel. Thus, it was believed that these stimulus-responsive, healing properties may eventually be used in various biomedical applications.

\section{Conflict of Interests}

The authors declare that there is no conflict of interests regarding the publication of this paper.

\section{Acknowledgments}

This paper is supported by "the Fundamental Research Funds for the Central Universities" and "Guangxi Key Laboratory of chemistry and Engineering of Forest Products Open Fund Project GXFC12-03.”

\section{References}

[1] P. Béguin and J.-P. Aubert, "The biological degradation of cellulose," FEMS Microbiology Reviews, vol. 13, no. 1, pp. 25-58, 1994.

[2] M. Knauf and M. Moniruzzaman, "Lignocellulosic biomass processing: a perspective," International Sugar Journal, vol. 106, no. 1263, pp. 147-150, 2004.

[3] B. D. Solomon, J. R. Barnes, and K. E. Halvorsen, "Grain and cellulosic ethanol: history, economics, and energy policy," Biomass and Bioenergy, vol. 31, no. 6, pp. 416-425, 2007.

[4] K. M. Rao, B. Mallikarjuna, K. S. V. Krishna Rao, M. N. Prabhakar, K. C. Rao, and M. C. S. Subha, "Preparation and characterization of $\mathrm{pH}$ sensitive poly(vinyl alcohol)/sodium carboxymethyl cellulose IPN microspheres for in vitro release studies of an anti-cancer drug," Polymer Bulletin, vol. 68, no. 7, pp. 1905-1919, 2012.

[5] C. Yang, C. Gao, Y. Wan, T. Tang, S. Zhang, and K. Dai, “Preparation and characterization of three-dimensional nanostructured macroporous bacterial cellulose/agarose scaffold for tissue engineering," Journal of Porous Materials, vol. 18, no. 5, pp. 545-552, 2011.

[6] N. Isobe, D.-S. Lee, Y.-J. Kwon et al., "Immobilization of protein on cellulose hydrogel," Cellulose, vol. 18, no. 5, pp. 1251-1256, 2011.

[7] S. Yan, J. Yin, L. Tang, and X. Chen, "Novel physically crosslinked hydrogels of carboxymethyl chitosan and cellulose ethers: structure and controlled drug release behavior," Journal of Applied Polymer Science, vol. 119, no. 4, pp. 2350-2358, 2011.

[8] X. Y. Qiu and S. W. Hu, "Smart materials based on cellulose: a review of the preparations, properties, and applications," Materials, vol. 6, no. 3, pp. 738-781, 2013.

[9] L. H. Fan, C. Tan, L. W. Wang et al., "Preparation, characterization and the effect of carboxymethylated chitosan-cellulose derivatives hydrogels on wound healin," Journal of Applied Polymer Science, vol. 128, no. 5, pp. 2789-2796, 2013.

[10] C. Chang, L. Zhang, J. Zhou, L. Zhang, and J. F. Kennedy, "Structure and properties of hydrogels prepared from cellulose in $\mathrm{NaOH} /$ urea aqueous solutions," Carbohydrate Polymers, vol. 82, no. 1, pp. 122-127, 2010. 
[11] Z. Wang, S. Liu, Y. Matsumoto, and S. Kuga, "Cellulose gel and aerogel from LiCl/DMSO solution,” Cellulose, vol. 19, no. 2, pp. 393-399, 2012.

[12] J. Zhang, J. Rong, W. Li, Z. Lin, and X. Zhang, "Preparation and characterization of bacterial cellulose/polyacrylamide hydrogel," Acta Polymerica Sinica, no. 6, pp. 602-607, 2011.

[13] C. Zhou, Q. Wu, Y. Yue, and Q. Zhang, "Application of rodshaped cellulose nanocrystals in polyacrylamide hydrogels," Journal of Colloid and Interface Science, vol. 353, no. 1, pp. 116123, 2011.

[14] A. L. Buyanov, I. V. Gofman, L. G. Revel'skaya, A. K. Khripunov, and A. A. Tkachenko, "Anisotropic swelling and mechanical behavior of composite bacterial cellulose-poly(acrylamide or acrylamide-sodium acrylate) hydrogels," Journal of the Mechanical Behavior of Biomedical Materials, vol. 3, no. 1, pp. 102-111, 2010.

[15] M. S. M. Eldin, H. M. El-Sherif, E. A. Soliman, A. A. Elzatahry, and A. M. Omer, "Polyacrylamide-grafted carboxymethyl cellulose: smart $\mathrm{pH}$-sensitive hydrogel for protein concentration," Journal of Applied Polymer Science, vol. 122, no. 1, pp. 469-479, 2011.

[16] R. Das, A. B. Panda, and S. Pal, "Synthesis and characterization of a novel polymeric hydrogel based on hydroxypropyl methyl cellulose grafted with polyacrylamide," Cellulose, vol. 19, no. 3, pp. 933-945, 2012.

[17] C. Chang, B. Duan, and L. Zhang, "Fabrication and characterization of novel macroporous cellulose-alginate hydrogels," Polymer, vol. 50, no. 23, pp. 5467-5473, 2009.

[18] C. Demitri, R. Del Sole, F. Scalera et al., "Novel superabsorbent cellulose-based hydrogels crosslinked with citric acid," Journal of Applied Polymer Science, vol. 110, no. 4, pp. 2453-2460, 2008.

[19] C. Chang, A. Lue, and L. Zhang, "Effects of crosslinking methods on structure and properties of cellulose/PVA hydrogels," Macromolecular Chemistry and Physics, vol. 209, no. 12, pp. 1266-1273, 2008.

[20] M. C. I. Mohd Amin, N. Ahmad, N. Halib, and I. Ahmad, "Synthesis and characterization of thermo- and $\mathrm{pH}$-responsive bacterial cellulose/acrylic acid hydrogels for drug delivery," Carbohydrate Polymers, vol. 88, no. 2, pp. 465-473, 2012.

[21] W. D. Li, J. H. Rong, Z. D. Lin, and X. J. Zhang, "Preparation and characterization of bacterial cellulose reinforced PVA/PVP hydrogels," Acta Polymerica Sinica, no. 4, pp. 357-364, 2012.

[22] R. Cha, Z. He, and Y. Ni, "Preparation and characterization of thermal/pH-sensitive hydrogel from carboxylated nanocrystalline cellulose," Carbohydrate Polymers, vol. 88, no. 2, pp. 713718, 2012.

[23] C. Chang, M. He, J. Zhou, and L. Zhang, "Swelling behaviors of pH- and salt-responsive cellulose-based hydrogels," Macromolecules, vol. 44, no. 6, pp. 1642-1648, 2011.

[24] W. Liu, Y. Liu, X. Hao et al., "Backbone-collapsed intra- and inter-molecular self-assembly of cellulose-based dense graft copolymer," Carbohydrate Polymers, vol. 88, no. 1, pp. 290-298, 2012.

[25] W. Yuan, J. Zhang, H. Zou, T. Shen, and J. Ren, "Amphiphilic ethyl cellulose brush polymers with mono and dual side chains: facile synthesis, self-assembly, and tunable temperature-pH responsivities," Polymer, vol. 53, no. 4, pp. 956-966, 2012.

[26] H. T. T. Phan, K. Zhu, A.-L. Kjøniksen, and B. Nyström, "Temperature-responsive self-assembly of charged and uncharged hydroxyethylcellulose-graft-poly(N-isopropylacrylamide) copolymer in aqueous solution," Colloid and Polymer Science, vol. 289, no. 9, pp. 993-1003, 2011.
[27] D. Wang, J. Tan, H. Kang et al., "Synthesis, self-assembly and drug release behaviors of $\mathrm{pH}$-responsive copolymers ethyl cellulose-graft-PDEAEMA through ATRP," Carbohydrate Polymers, vol. 84, no. 1, pp. 195-202, 2011.

[28] M. Bagheri and S. Shateri, "Thermosensitive nanosized micelles from cholesteryl-modified hydroxypropyl cellulose as a novel carrier of hydrophobic drugs," Iranian Polymer Journal, vol. 21, no. 6, pp. 365-373, 2012.

[29] J. Wan, Z. Hu, W. Chen, H. Chen, and Z. Yu, "Synthesis and application on enrichment of metal ions of cellulose fibre grafted $\beta$-cyclodextrin," Acta Polymerica Sinica, no. 4, pp. 566$572,2004$.

[30] E. Kosecka-Judin, M. Wesolowski, and D. Paukszta, "Pattern recognition methods as supplementary techniques for identification of salicylamide-cyclodextrins inclusion complexes," Central European Journal of Chemistry, vol. 10, no. 5, pp. 15341546, 2012.

[31] C. Volobuef, C. M. Moraes, L. A. S. Nunes et al., "Sufentanil-2hydroxypropyl- beta-cyclodextrin inclusion complex for pain treatment: physicochemical, cytotoxicity, and pharmacological evaluation," Journal of Pharmaceutical Sciences, vol. 101, no. 10, pp. 3698-3707, 2012.

[32] M. J. Arias-Blanco, J. R. Moyano, J. I. Perez-Martinez, and J. M. Gines, "Study of the inclusion of gliclazide in $\alpha$-cyclodextrin," Journal of Pharmaceutical and Biomedical Analysis, vol. 18, no. 1-2, pp. 275-279, 1998.

[33] F. Giordano, C. Novak, and J. R. Moyano, "Thermal analysis of cyclodextrins and their inclusion compounds," Thermochimica Acta, vol. 380, no. 2, pp. 123-151, 2001.

[34] B. Szafran and J. Pawlaczyk, "Preparation and characterization of the $\beta$-cyclodextrin inclusion complex with sulfafurazole," Journal of Inclusion Phenomena and Molecular Recognition in Chemistry, vol. 23, no. 4, pp. 277-288, 1996.

[35] A. Harada and S. Takahashi, "Preparation and properties of cyclodextrin-ferrocene inclusion complexes," Journal of the Chemical Society, Chemical Communications, no. 10, pp. 645646, 1984.

[36] T. Matsue, D. H. Evans, T. Osa, and N. Kobayashi, "Electrontransfer reactions associated with host-guest complexation: oxidation of ferrocenecarboxylic acid in the presence of $\beta$ cyclodextrin," Journal of the American Chemical Society, vol. 107, no. 12, pp. 3411-3417, 1985.

[37] M. Nakahata, Y. Takashima, H. Yamaguchi, and A. Harada, "Redox-responsive self-healing materials formed from hostguest polymers," Nature Communications, vol. 2, no. 1, article 511, 2011.

[38] A. Nakayama, A. Kakugo, J. P. Gong et al., "High mechanical strength double-network hydrogel with bacterial cellulose," Advanced Functional Materials, vol. 14, no. 11, pp. 1124-1128, 2004.

[39] A. Sannino, C. Demitri, and M. Madaghiele, "Biodegradable cellulose-based hydrogels: design and applications," Materials, vol. 2, no. 2, pp. 353-373, 2009. 

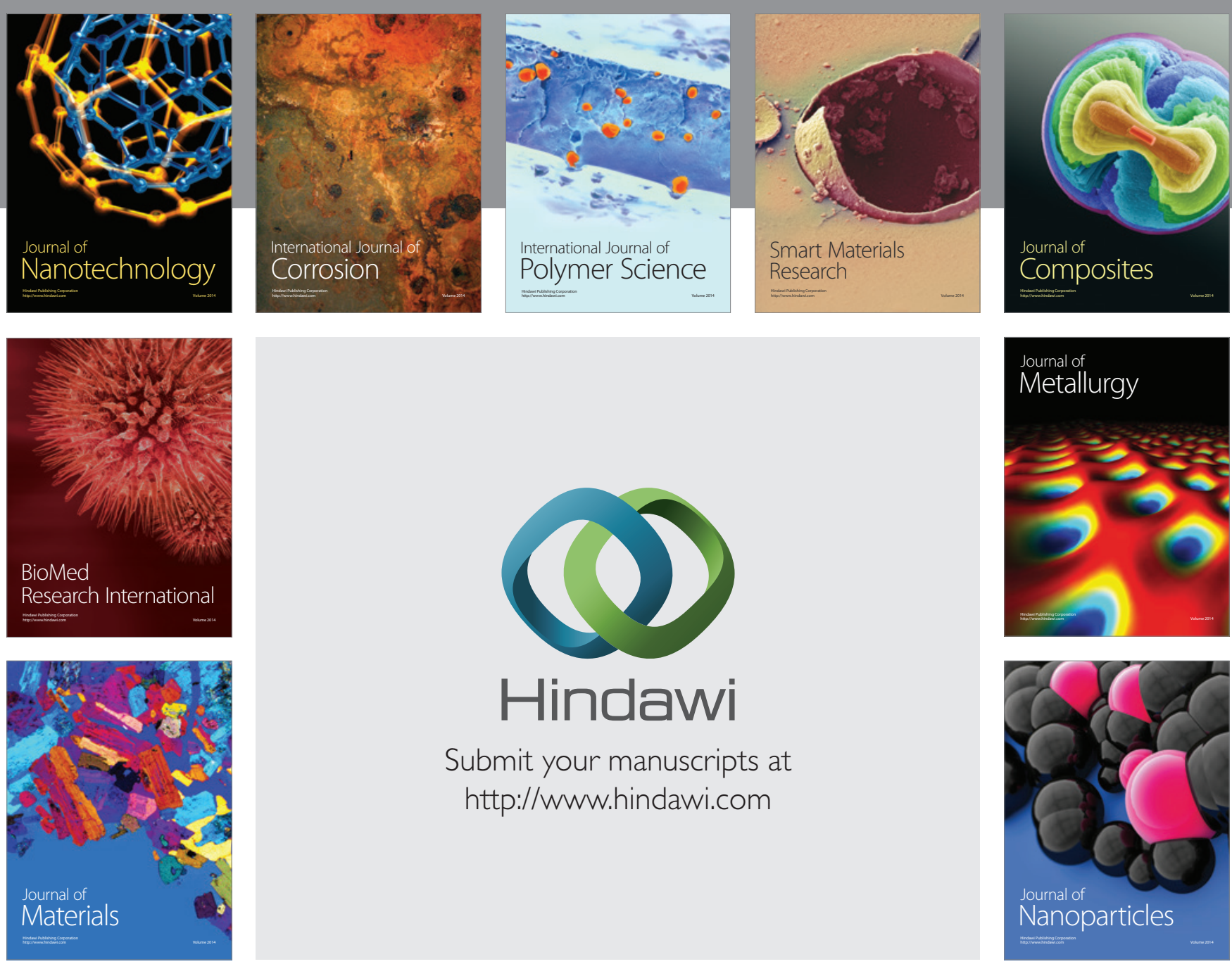

Submit your manuscripts at http://www.hindawi.com
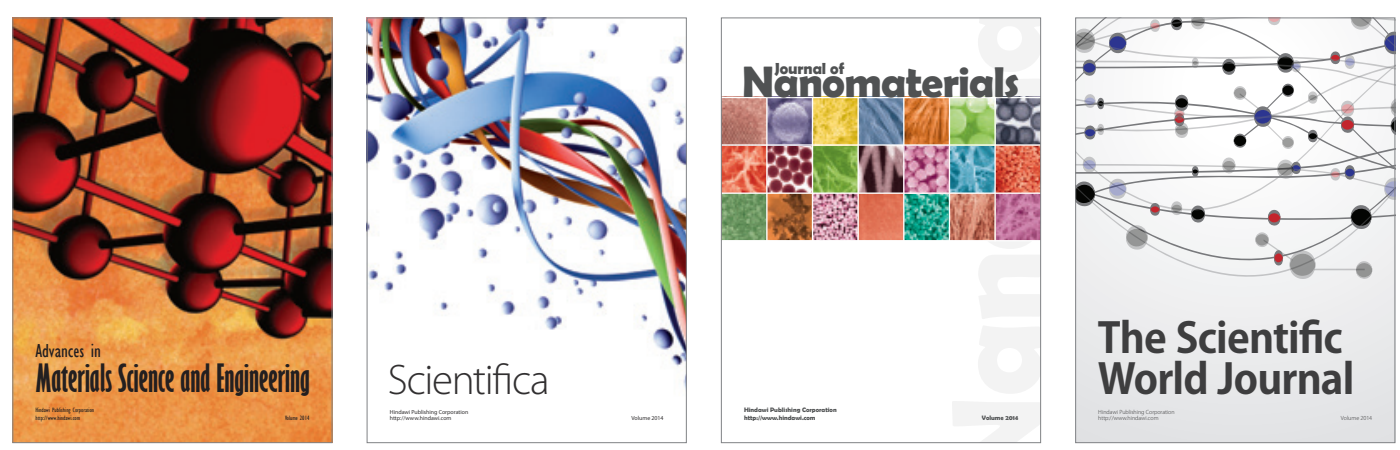

\section{The Scientific World Journal}
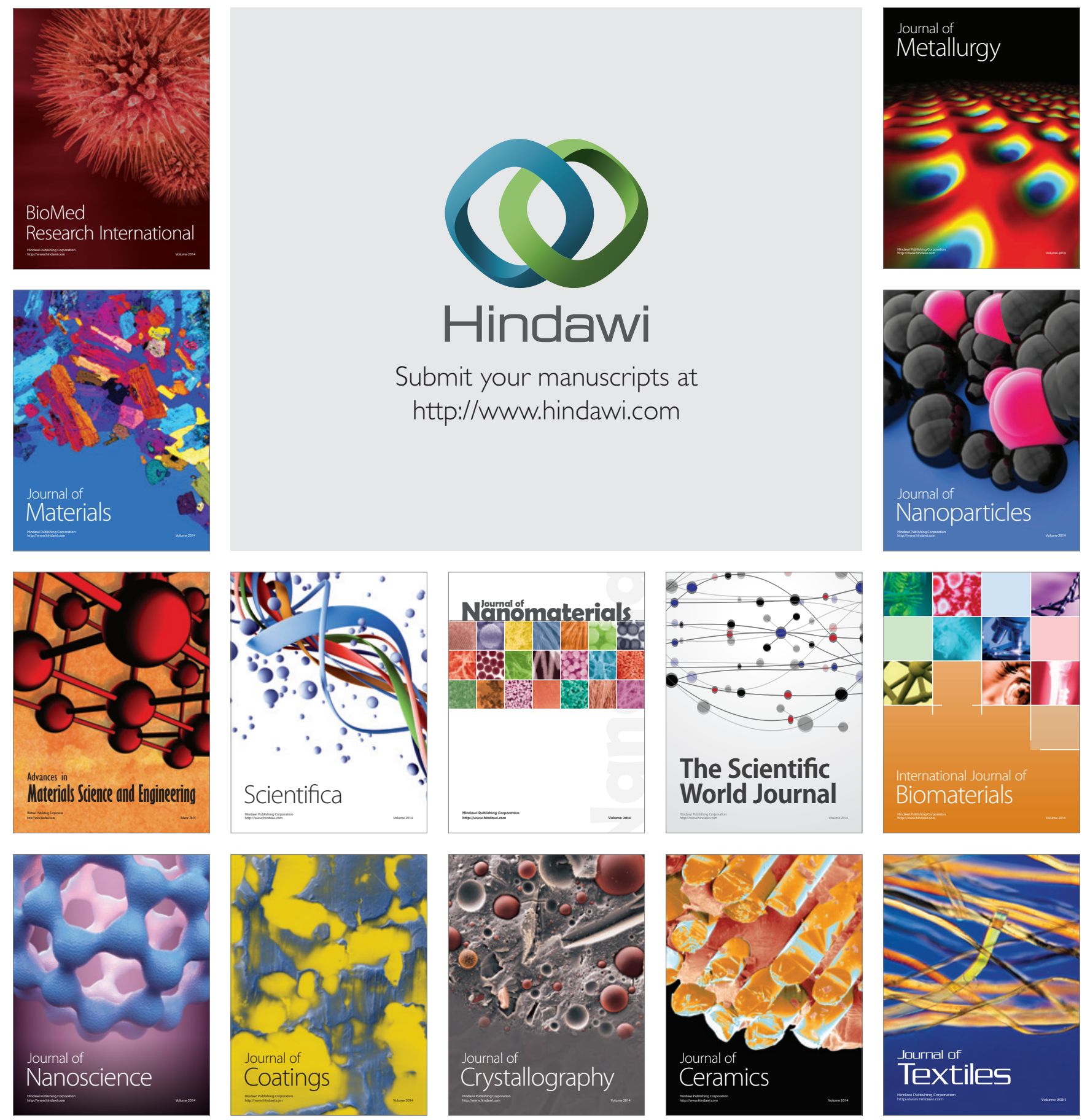